\title{
lot Based Smart Switch Board Technology For Smart Home
}

\author{
A. Ranjith Kumar \\ Assistant Professor, \\ School of Computer Science and Engineering, \\ Lovely Professional University, Phagwara, India \\ ranjith.26108@1pu.co.in \\ Karanvir Singh \\ Research Scholar, \\ School of Computer Science and Engineering, \\ Lovely Professional University, Phagwara, India \\ kvsjxd@gmail.com
}

\begin{abstract}
:
To overcome the limitations of the traditional switch board, a new system equipped with latest technologies has been developed as Smart Switch Board and which is the smarter version compared to traditional Switch Boards. An IOT based switching device has been developed and which to be controlled via Blynk application. This smart device is very useful for handicapped persons. The smart switch meter has been used to control the smaller areas if it is connected through the router. It makes the system more secured as it is working through router's IP address. Physical contact is not required to operate; it ensures reliability and safety from fire accidents. The Hardware component are quite cheap and easy to replace.
\end{abstract}

\section{Keywords:}

\section{INTRODUCTION:}

Home automation or Smart Homes (also referred to as domotics) are often described as a presentation of innovation within the house environment to supply convenience, solace, security, and energy proficiency to its tenants. Adding insight to home climate can give expanded personal satisfaction to the older and disabled people that might in any case require guardians or institutional consideration. There has been a big increase in home mechanization in ongoing years thanks to higher moderateness and progression in smartphones and tablets which permits tremendous availability. With the presentation of the web of Things, the examination and execution of home automation are becoming more well known.

This involves the necessity of presenting shrewd exchanging innovation. This venture addresses "Smart Board" is that the smarter adaptation to the normal switch circuit. This change is an in the field of IoT which empowers which is being controlled through Blynk application to savvy gadgets and this element would turn out to be extremely helpful for the old and crippled people. The brilliant smart board is developed for the accessible way to assist the system. Individuals can work and control their apparatuses through their smartphones from any place in the world. In this manner, they will also save energy.

It is like a kind of system which is being developed using a combination of hardware and for operating that we will be using the software as a work, this idea of development for the smart system for the comfort of the people and it will be the best thing for the people who are 
differently-abled as it will be very easy to use this kind of machine being developed by a single click of the hand.

\section{Basic Structure of Immerse System}

Sensor device - It can be called software and hardware which takes input from the outside world as physical and converts it as in a machine term which can be either read by human or machine.

A TO D Converter - Associate in Nursing data converter is used for changing the analog signal into the digital signal.

Mini Processor \& ASIC device - It is used for sending input and storing the output into a set of devices for further use.

D To A device - It is used to transfer information from digital or convert it into analog from digital.

\section{PROBLEM STATEMENT}

The modern era is usually seen as a good era to live in where every old gadget is becoming obsolete and is being changed with newer and improved features. This idea for changing and improving the old gadgets into modern ones with new features is creating a new trend for the people to improve the old gadgets so that one can control and operate them even when we are not at home. In this fashion, they'll additionally save energy. So the main problem behind this creation is that as nowadays everything around this world is being developed into the latest thing so the previous use of the home electric gadgets are controlled by physical means and it must require someone to switch on or off that particular thing but nowadays that is only becoming a very big problem as for the people who cannot perform physical activity and they cannot move or whether they don't wish to move, so form them and for all people in or around this globe will be going to get beneficial by our project or the machine which we are creating, In the market, there are many latest things are available but there cost are very high which will not be able and can be afforded by everyone so here comes our idea for creating this new tech machine.

\subsection{SCOPE OF PROJECT}

The scope for this project will be very wide as the combination of software and hardware which we are developing will not be used as a wish or fashion rather than this will be very helpful to each and every person. Its scope is very wide and in many numbers of sectors, this will remove physical contact barriers when it will get used as this machine is accessible using a Wi-Fi access point, so the people will be able to control their gadgets very easily. It is very easy to install anywhere and can be repaired easily. As opposed to people who may think this project will be hard to use, in reality, it is easy to set up and use without investing a lot of money. 
The IoT sector is providing benefits to the consumers by their introduction of new and impressive features which is providing users with happiness among various cultures.

\section{Applications of IoT}

\section{Consumer Application(s)}

- Smart home for care of elderly

\section{Commercial Applications}

- Medical and Healthcare automation

- Transportation system automation

- V2X communication

- Home and enterprise automation

\section{Industrial Application(s)}

- Manufacturing farm automation

\section{Infrastructure Application(s)}

- Energy management and analysis automation in metropolitan cities

\section{EXISTING SYSTEM}

\subsection{Introduction}

There are many Smart Switch Boards available within the market but none of them are giving the customer the satisfaction they have; old devices are replaced with smart ones. As the previous circuit has to be changed when merging it with new technology. The main goal of such upgrades is the user's satisfaction with new features provided by the technology. Along with providing users with happiness, there is also a focus on saving energy and at the same time making them operate faster. These modern gadgets improve user's life by providing them with more ease since these gadgets connect wirelessly to Wi-Fi and can be controlled over WAN using an application on a smartphone which can be anywhere in the world.

\subsection{Existing Software}

As time is going fast and new gadgets have been coming up, they have been replacing older ones in the market. This process also includes innovation. Currently, there are many gadgets in the market that underestimate the needs of people, they fail to satisfy the people in need which can be the reason why some people might not be able to trust the latest tech and fashion being developed today, the products which give trust to people can clearly fail to provide people's need.

Present Products which have comparative usefulness have a tremendous measure of cost, which are very exorbitant costs for the people to utilize that item. So, it likewise inspires us to figure on a modest yet viable keen switchboard for people.

\subsection{What Needs to Be Developed in the New System}


Our system will provide advancement within the field of IoT and within home automation and in many fields like smart home, elder care, medical and health care, building, and residential automation. Smart switchboards are going to be created considering the available technologies to assist people. People can operate and control their appliances through their smartphones from anywhere in the world. In this manner, they will also save energy. So, looking into the people's mind we are now ready to introduce our latest trend with the combination of hardware and software, the new machine can be called as power switch machine.

As the update is being done towards every field of use so that why this system must need to come into the picture for everyone. This will be the combination of smart tech devices which are being developed for providing people with their needs. This device is also targeted towards old and weakened individuals.

This set of components will contain the safety measures for scenarios where electric board at someone's home catches fire by accident then the owner will immediately get the report and info regarding any harm which is being caused to their electric gadget Gear parts used in savvy switchboard are modest and direct to trade.

\section{DESIGN}

\subsection{System Design}

The basic correspondence occurs between the taking care of unit for the exchange need to take place in relation to the system. That contains no GPIO microdevices that are operated by the customer at execution. As the getting ready system contains combination with that, that supports the change for talk as of customer that exhibits for the location containing switch bring into picture into the applications and when we will start powering on or off switch, the working action takes place that is Node MCU start reading the signal from the input and then transfers it to the relay module for next step. Then exchange gets to sign it trips after a certain proportion of minutes (the 1000ms) and the bulb or weight that is related to the hand-off is killed. Likewise, when the bulb is powered from the android application, Node measures it, and then it passes the signal through microunit for further state. When the main unit gets the signal from the previous one it starts doing its work and the system gets to work within a second which comes really fast. And from that end as like the signal is being given or provided the owners for the signal wait to respond and as they touch the signal, they got to complete the task.

\subsection{Design Notations}




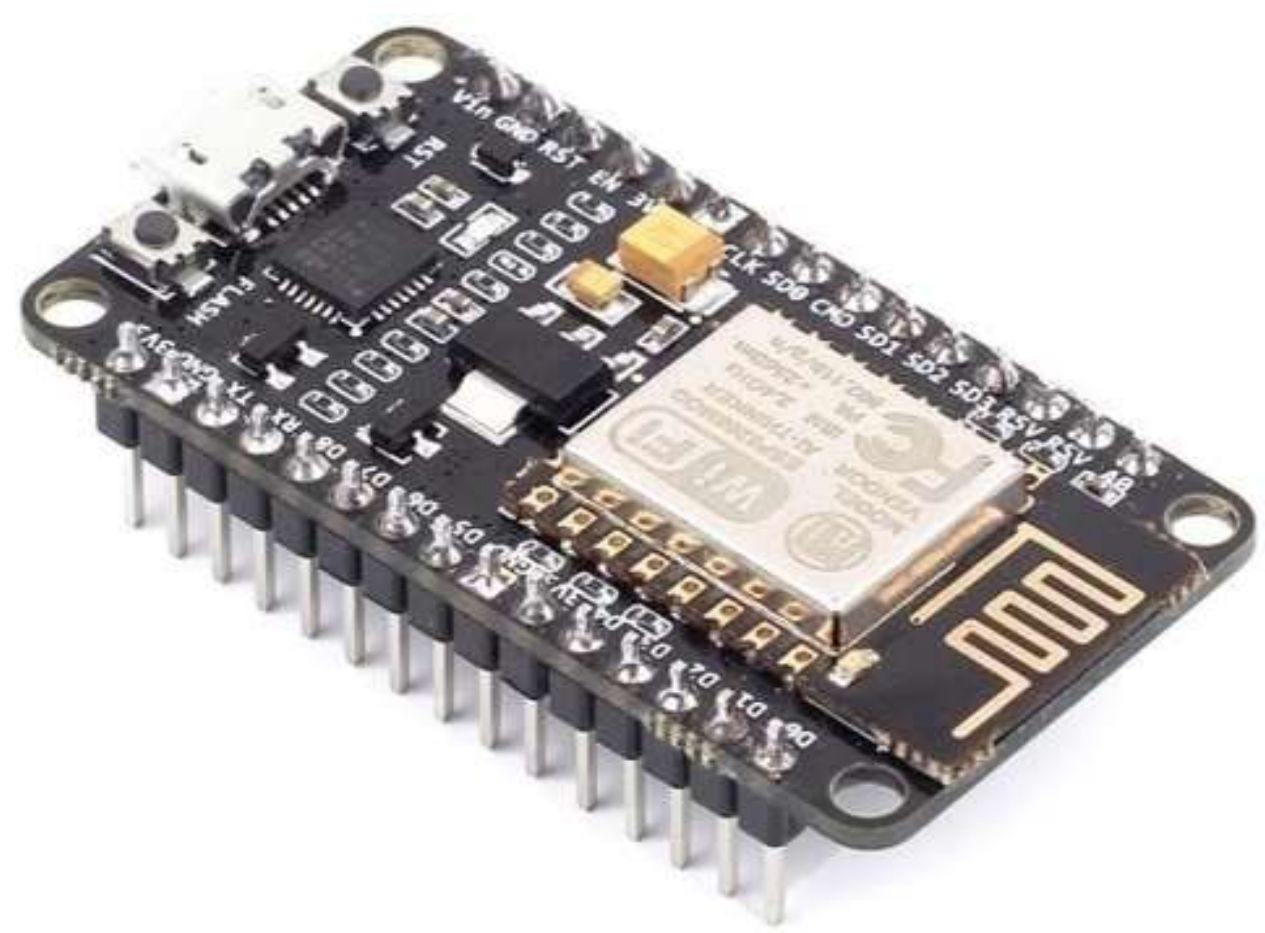

Fig: 8 - Node MCU from outer side

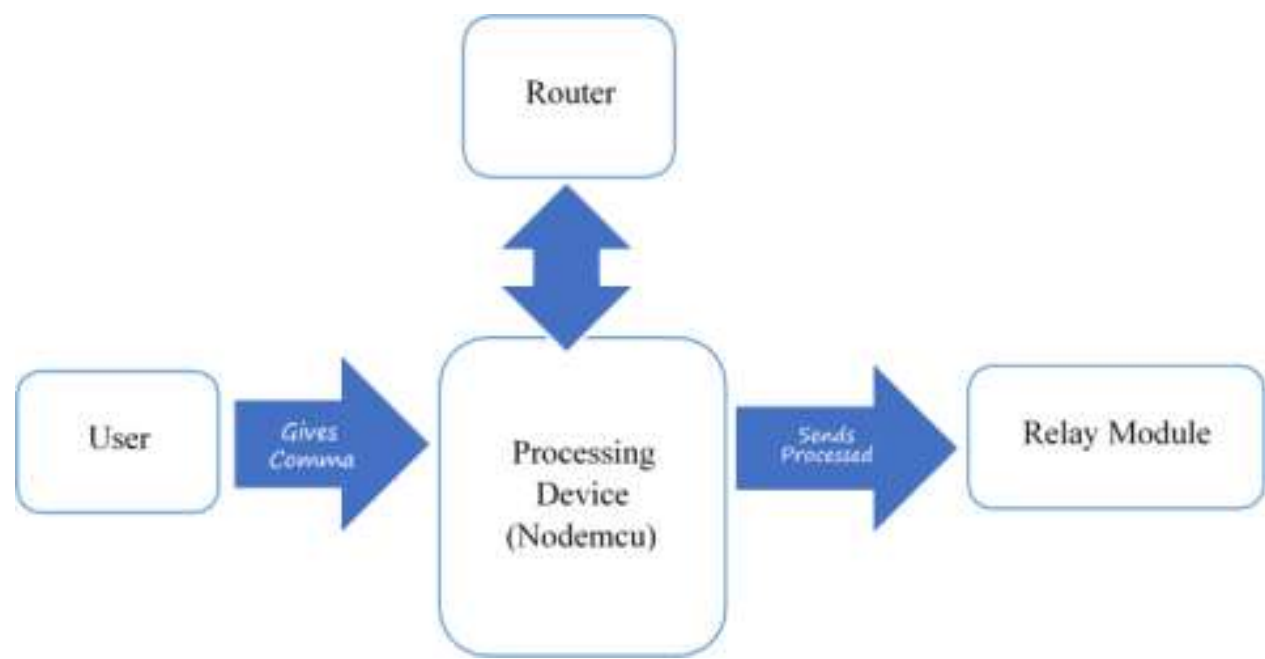

Fig: 9 - Processing unit

\section{Input power for internal component}

To offer the capability to the inner segments of the framework, we utilized AC to DC transformation by means that of an electrical device that is a normal interaction. In spite of the very fact that the electrical device is cumbersome and weighty but it's improved than the opposite quite amendment technique that is that the essential justification selecting electrical device. Moreover, the electrical device isn't troublesome to execute, usually economical, utterly disconnected, and supposed for all forms of burdens. The schematic define of the amendment circuit is given beneath:

\section{Level 0 DFD:}




\section{Relay \& Node Mcu}

The preparing gadget which is getting used will be Node MCU. That is hardware used in the IoT field. The thing which it takes into action will be [ESP-12] and will get execute to [ESP8266]. Codes are regularly utilized like Arduino, however intelligently bundle-like. The system which is the main reason for the networking field which works with designers composing a piece for the coding can happen on NODE.JS format by using a device MCU and extraordinarily accelerate the development of new gadgets.

Relay is an associate in Nursing electrically worked switch. Distinctive hand-off uses an associate in Nursing magnet to without ambiguity work a switch, regardless of different operating pointers is what is more used, as solid-state moves. Move AR is used wherever it's essential to manage a circuit by the associate in Nursing different low-power sign, or a number of circuits need to be duty-bound by one sign. A transfer which will manage little energy expected for unquestionable management of electrical rotor or numerous burdens for thought as the system moves management with the new system, rather employing for a conductor for functioning commercialism.

The benefit of interfacing to the switch must be entire framework is gotten in light-weight of the actual fact that the framework cannot be gotten to by untouchables. Moreover, the Node MCU is related to the transfer module and on these lines, the hand-off module is employed for interfacing a whole bunch. For this purpose, once the transfers area unit goes off, a whole bunch related to them gets modified from the framework. On the contrary, hand, turning off transfers interface a whole bunch with the framework.
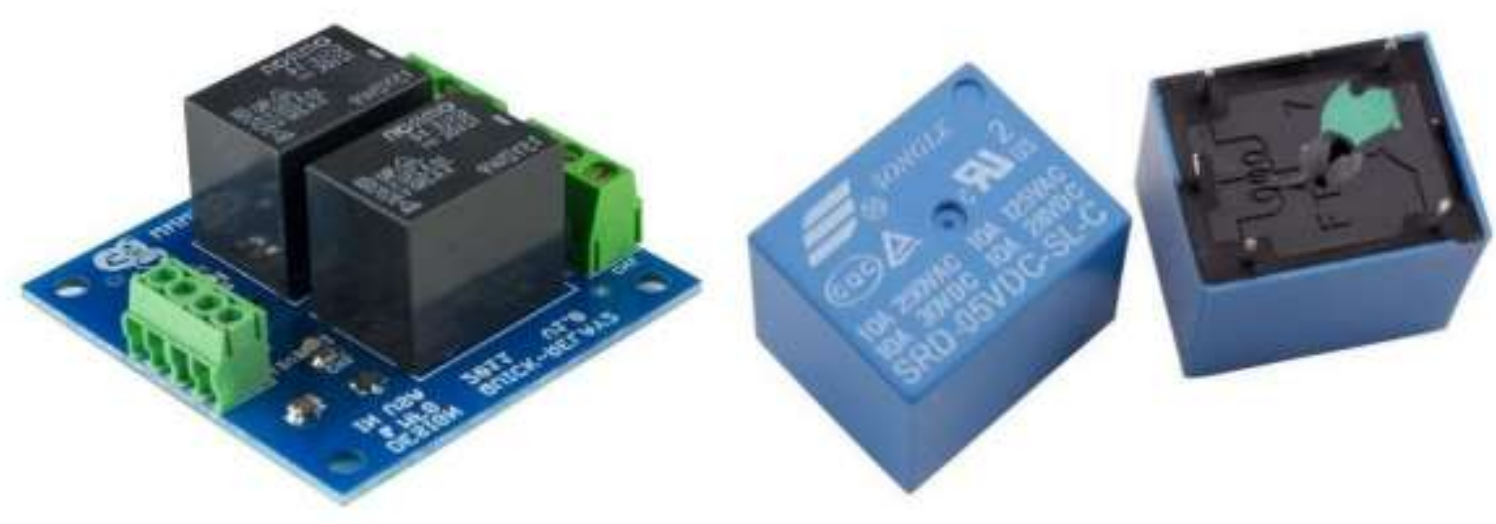

Fig 11: Schematic Figure of Relay

\section{Level 1 DFD:}

Initially, the start of the process provide from the facility supply is modified over to dc (here five potential unit DC with one amp DC current) through an inward circuit comprising the electrical device, controller, electrical condenser, and totally different 
segments. Here, the amendment technique used is customary and really solid. The modified over five potential units DC is employed to supply capability to the Node MCU and to Relays. The Node MCU is related to the switch and therefore the switch is related to the online. Consequently, it tends to be gotten to by the science address of the switch for playacting exchanging activity with the heap. One good thing about interfacing with the switch is that the complete framework is gotten because the framework cannot be gotten to by outcasts. Moreover, the Node MCU is related to the transfer module and therefore the hand-off module is employed for interfacing the lots. For this purpose, once the transfers area unit is set out, the lots related to them get turned aloof from the framework. Then again, turning off transfers associate the lots with the framework.

\section{Level 2 DFD:}

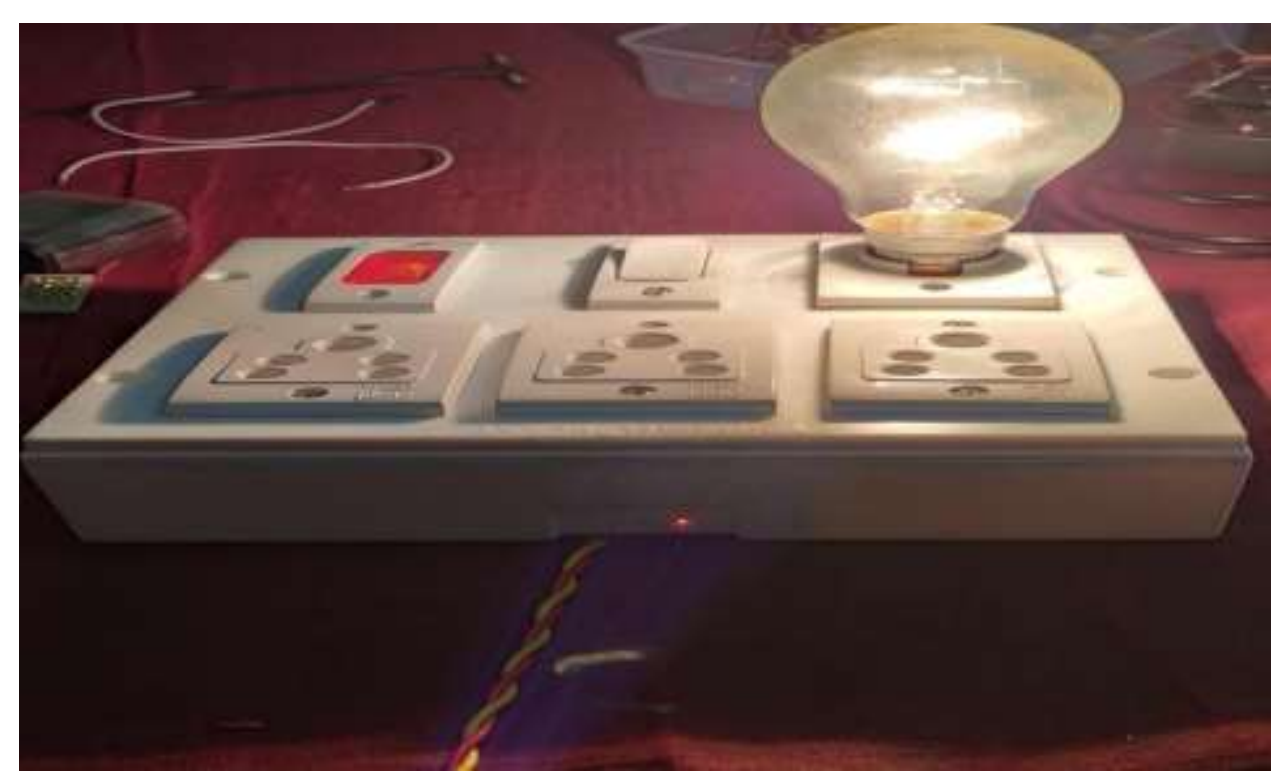

Fig: 13 - Final View of Smart Switch Board

\subsection{Flow Chart}

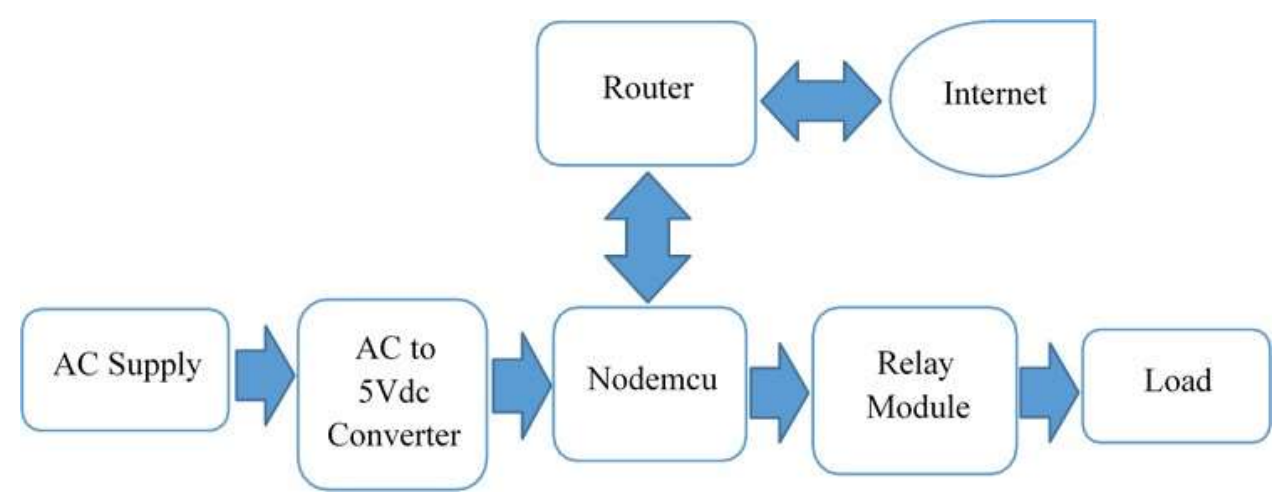

Fig: 14 - Flow Chart of the Circuit 
The above block diagram shows the framework engineering of our venture. First and foremost, the cooling system offer by the ability supply is modified over to DC (here 5 volts DC with 1 amp DC current) through an internal circuit comprising an electrical device, controller, electrical condenser, and completely different segments. Here, the transformation technique utilized is standard and really dependable. The modified over 5 volts DC is employed to supply capability to the Node MCU and to Relays. The Node MCU is related to the switch and afterward, the switch is related to the online. Afterward, its area unit got to by the scientific discipline to the location of the switch for doing arts exchanging activity that contains heap. The benefit of interfacing to the switch must be entire framework is gotten in light-weight of the actual fact that the framework cannot be gotten to by untouchables. Moreover, the Node MCU is related to the transfer module and on these lines, the hand-off module is employed for interfacing a whole bunch. For this purpose, once the transfers area unit goes off, a whole bunch related to them gets modified from the framework. On the contrary, hand, turning off transfers interface a whole bunch with the framework.

\subsection{Implementation of Project}

When the 5-volt AC supply is being given to the circuit with the combination of the DC circuit to the transformer installed inside the system that contains no GPIO microdevices that are operated by the customer at execution. As the getting ready system contains combination with that, that supports the change for talk as of customer that exhibits for the location containing switch bring into picture into the applications and when we will start powering on or off switch, the working action takes place that is Node MCU start reading the signal from the input and then transfers it to the relay module for next step. Then exchange gets to sign it trips after a certain proportion of minutes (the $1000 \mathrm{~ms}$ ) and the bulb or weight that is related to the hand-off is killed. Likewise, when the bulb is powered from the android application, Node measures it, and then it passes the signal through microunit for further state. When the main unit gets the signal from the previous one it starts doing its work and the system gets to work within a second which comes really fast. And from that end as like the signal is being given or provided the owners for the signal wait to respond and as they touch the signal, they got to complete the task. Transfers are set off, the heaps associated with them get turned off from the framework. As demonstrated in the beneath char. The basic correspondence occurs between the taking care of unit for the exchange need to take place in relation to the system. That contains no GPIO microdevices that are operated by the customer at execution. As the getting ready system contains combination with that, that supports the change for talk as of customer that exhibits for the location containing switch bring into picture into the applications and when we will start powering on or off switch, the working action takes place that is Node MCU start reading the signal from the input and then transfers it to the relay module for next step. Then exchange gets to sign it trips after a certain proportion of minutes (the $1000 \mathrm{~ms}$ ) and the bulb or weight that is related to the hand-off is killed. Likewise, when the bulb is powered from the android application, the node measures it, and then it passes the signal through microunit for further state. When the main unit gets the signal from the previous one it starts doing its work and the system gets to work within a second which comes really fast. And from that end as like the signal is being given or provided the 
owners for the signal wait to respond and as they touch the signal, they got to complete the task.

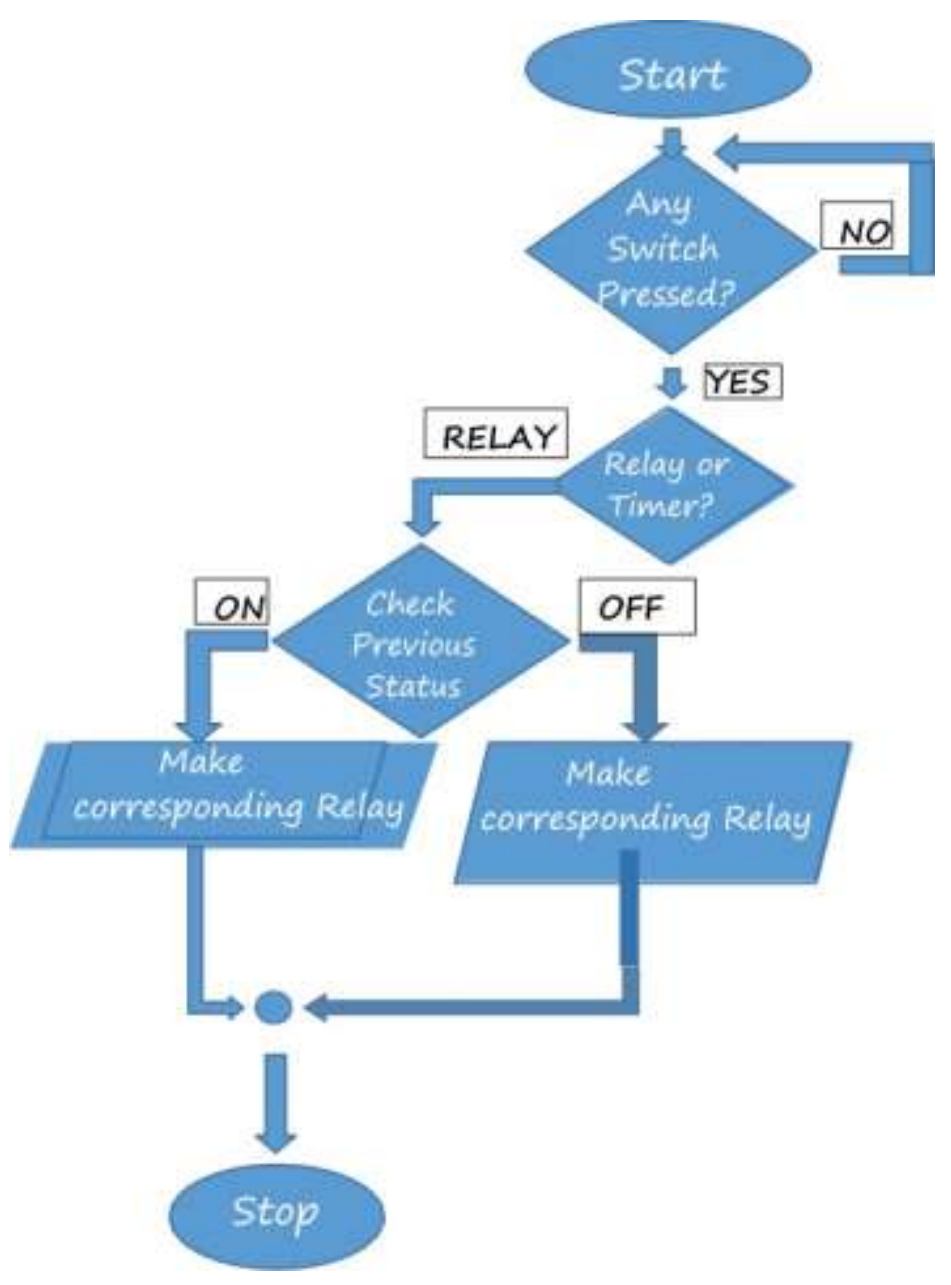

Fig: 16 - Flow Chart

\subsubsection{Application}

\section{SOFTWARE (Blynk Application)}

The software package for dominant the good Switch circuit is Blynk Application created basically as for humanoid system that is that the biggest introduced concept for the versatile working framework. The user interface for this application contains basic and passionate about direct management utilizing show contact signals. Essentially slightly on the catches apparent within the screen of the phone is spare to start an order. The first intention of the applying is to figure our savvy exchanging board remotely utilizing our phone by utilizing the Wi-Fi put in on the telephone's instrumentation. 


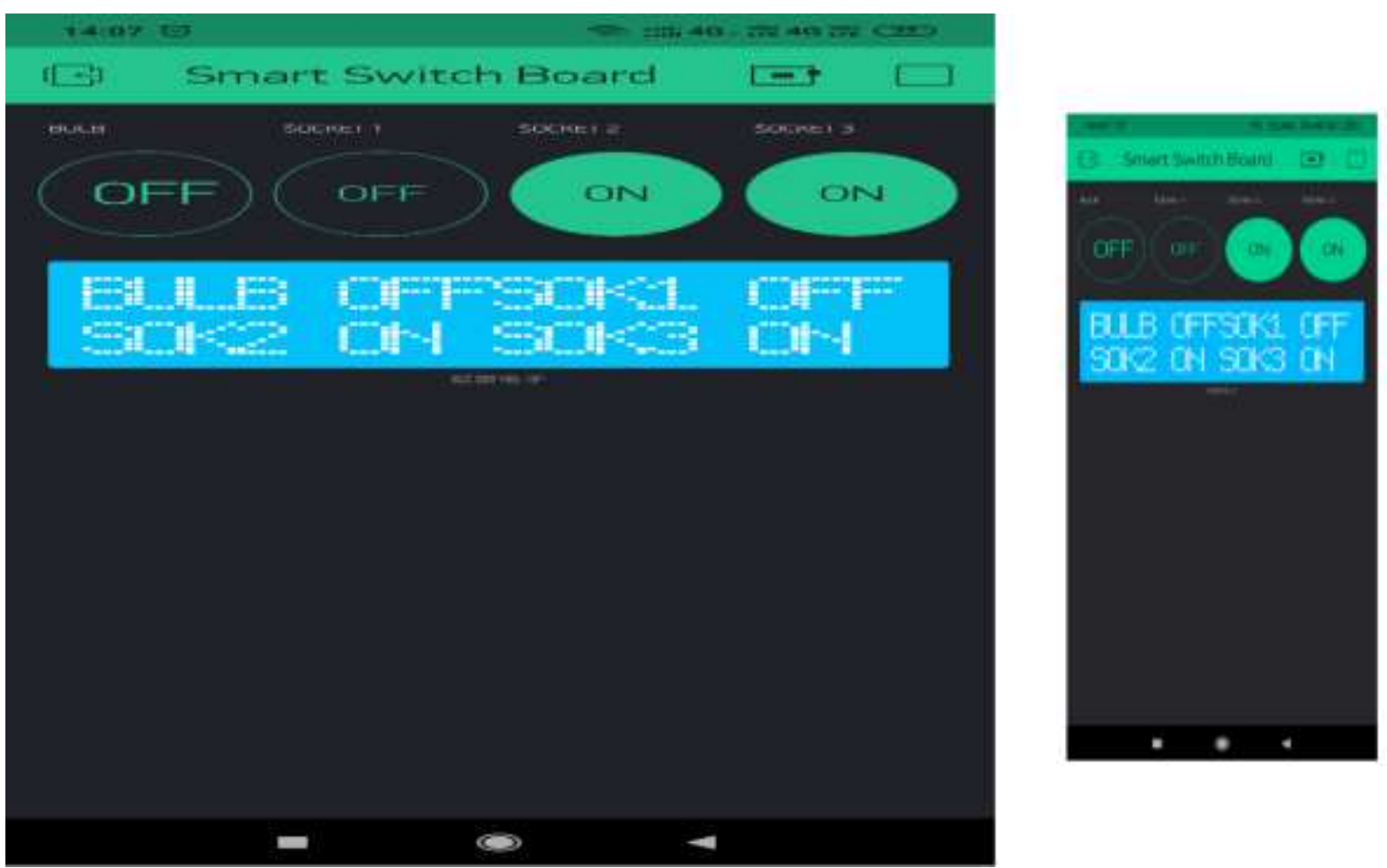

Fig:17 User Interface (UI) for user at Blynk App

\section{PROJECT LEGACY}

\subsection{Current status of the project}

Our device is an IoT Based Smart Switch Board that is currently in a fully functional state and running smoothly. Data is being stored and accessed from the database properly with no redundancy, inconsistency, and irregularity. The website is available for users $24 / 7$ without any error.

\subsection{Remaining areas of concern}

Although the project is fully functional, many functionalities can be added in this project. Following are the functionalities that we plan to add in the future:

- We have planned to add direct monitoring features for the organization. The organization has to set up a high-speed Wi-Fi system so that they can monitor the home system directly. So, in this, we are planning to give extra control to the owner so that he can monitor performance easily.

- We plan to add more nodes so that we can increase the efficiency of the home automated system. So, as we plan to add more nodes so that we can decrease the packet loss of the system

\subsection{Details for the advance system}

Below flow chart will show us the detailed information for advance system to developed. 


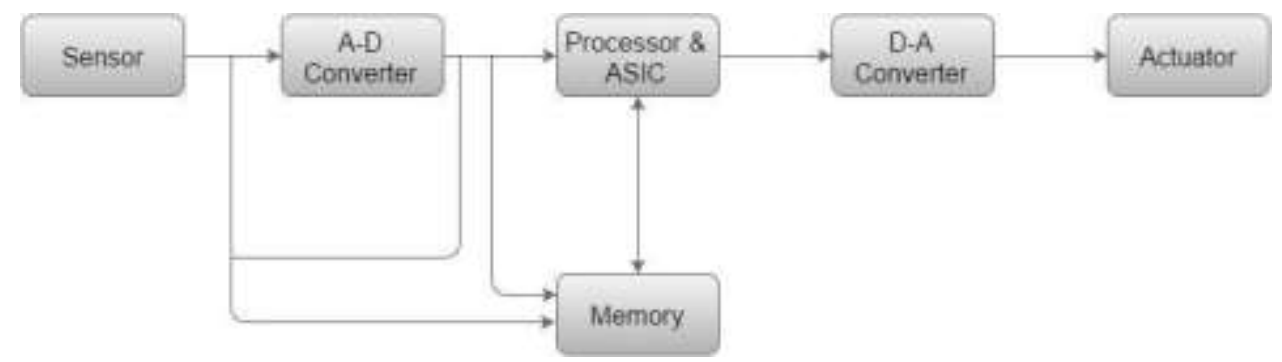

Fig: 18 - Structural Diagram of Embedded System

\subsection{Processing device}

\section{Node MCU}

The preparing gadget which is getting used will be Node MCU. That is hardware used in the IoT field. The thing which it takes into action will be [ESP-12] and will get execute to [ESP8266]. Codes are regularly utilized like Arduino, however intelligently bundle-like. The system which is 42 the main reason for the networking field which works with designers composing a piece for the coding can happen on NODE.JS format by using a device MCU and extraordinarily accelerate the development of new things. anyway because of a handful of key options nodemcu is employed in our endeavor that consolidates insignificant value, instinct, straightforwardness, snappiness, Wi-Fi-engage work, etc. 

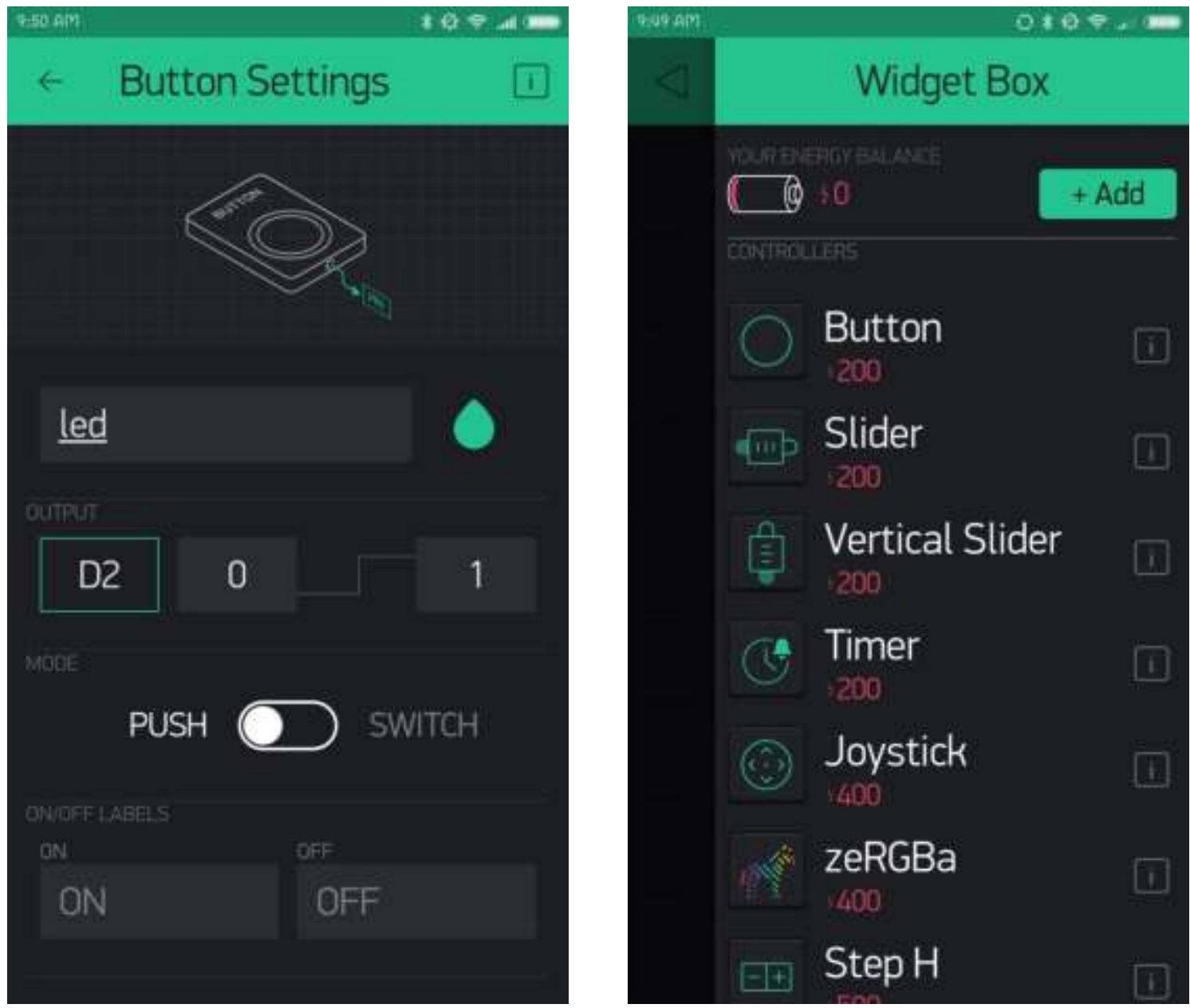


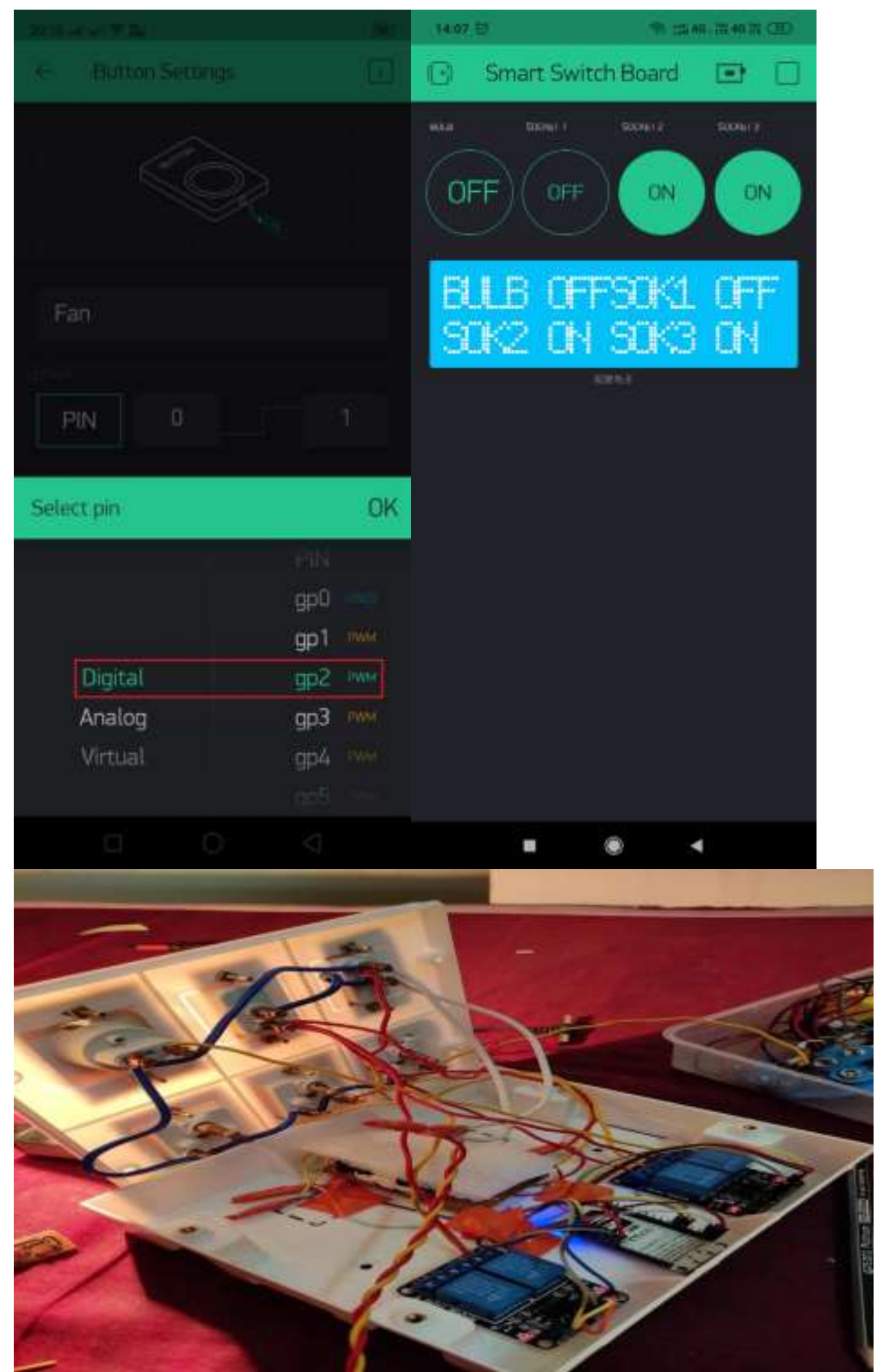

\section{BIBLIOGRAPHY}

[1] OKIoT: Trade off analysis of smart speaker architecture on open knowledge IoT projectVictor T. Hayashi, Reginaldo Arakaki, Wilson V. Ruggiero December 2020 
Page 100310

[2] A ZigBee Intrusion Detection System for IoT using Secure and Efficient Data CollectionFal Sadikin, Ton van Deursen, Sandeep Kumar December 2020 Page 100306

[3] Himesh, S. Digital revolution and Big Data: A new change in field of iot . CAB Rev. 2018, 13, 1-7..

[4] N. Gligoric et al., "Smartdev: IoT Product based Passport for Circular Economy depend on Printed Sensors and Unique Item-Level Identifiers," Sensors, vol. 19, no. 3, 2019.

[5] Agbehadji I. E, Millham R. C, Fong S. J, Jung J. J, Bui K. H. N, \& Abayomi A, (2019), "multi-stage steering convention for energy improvement in remote sensor in systems administration. In Worldwide Conference on Soft Computing and Data Science (pp. 223-238)"-Springer,

Singapore.

[6] Javed Mehedi Shamrat, Naeemul Islam Nobel, Zarine Tasnim and Moe. "An Automatic sensor Detection system to Prevent Passenger vehichles Accidents Due to Overweight". Fifthh International Conference on IoT (IoT'19).

[7] Tarhani M, Kavian Y. S, and Siavoshi S, (2014), "SECH: Scalable energy for making productive Robotization progressive system convention in remote sensor organizations"- IEEE Sensors Journal, 14(11), 3944-3954

[8] Ando H, Barolli L, Durresi A, Xhafa F, \& Koyama A, (2010), “A wise fluffy based bunch head determination framework for remote sensor organizations and its presentation assessment"- In 2010 thirteenth International Conference on NetworkBased Information Systems (pp. 55-61). IEEE

[9] Al-Hubaishi M, Çeken C, \& Al-Shaikhli A, (2019), "A novel energy-aware routing mechanism for SDN-enabled WSAN"-International Journal of Communication Systems, 32(17), e3724.

[10] F.M. Javed Mehedi Shamrat, Naeemul Islam Nobel, and Razu Ahmed "Implementation of Smart

Embedded Systems for Computational Intelligence" in Computational Intelligence, Security and IoT (ICCIIIT), Volume 1192, Article Number: 35, 2020 pp. : 357-370, DOI: https://doi.org/10.1007/978- 